\title{
Correção cirúrgica do estrabismo horizontal em portado- res de alta miopia
}

\author{
Surgical correction of horizontal strabismus in high myopia patients
}

\author{
Fábio Ejzenbaum ${ }^{1}$ \\ Mauro Goldchmit ${ }^{2}$ \\ Carlos R.Souza-Dias ${ }^{3}$ \\ Marcelo F. Gaal Vadas ${ }^{4}$
}

\begin{tabular}{|c|}
\hline RESUMO \\
\hline $\begin{array}{l}\text { Objetivo: Análise dos resultados cirúrgicos da correção dos estrabismos } \\
\text { horizontais em portadores de alta miopia, em pacientes do Departamento } \\
\text { de Oftalmologia da Santa Casa de Misericórdia de São Paulo. Métodos: } \\
\text { Foram estudados os prontuários de } 24 \text { pacientes esotrópicos e } 17 \\
\text { exotrópicos, portadores de miopia maior que } 6,00 \mathrm{DE} \text { operados para } \\
\text { correção do estrabismo. Consideramos como bons resultados cirúrgicos } \\
\text { desvios residuais entre esotropia e exotropia de } 10 \Delta \text {. Resultados: Obser- } \\
\text { vou-se grande incidência de maus resultados entre os pacientes } \\
\text { esotrópicos altos míopes. Conclusão: Concluímos que existe uma ten- } \\
\text { dência a piores resultados cirúrgicos nos pacientes esotrópicos com } \\
\text { miopia maior que }-6,00 \mathrm{DE} \text {, em comparação com esotrópicos com erro } \\
\text { refrativo entre- }-75 \mathrm{DE} \text { e }+3,50 \mathrm{DE} \text {. }\end{array}$ \\
\hline
\end{tabular}

Descritores: Estrabismo/cirurgia; Miopia; Exotropia; Esotropia; Cirurgia

\footnotetext{
Médico do curso de aperfeiçoamento do Departamento de Oftalmologia da Santa Casa de São Paulo.

Doutor em Oftalmologia pela Universidade Federal de São Paulo - Escola Paulista de Medicina; chefe do setor de Motilidade Extrínseca Ocular do Departamento de Oftalmologia da Universidade de Santo Amaro e médico assistente voluntário do Departamento de Oftalmologia da Santa Casa de São Paulo.

${ }^{3}$ Professor Livre Docente pela Universidade Federal de São Paulo - Escola Paulista de Medicina e Professor Titular da Faculdade de Ciências Médicas da Santa Casa de São Paulo.

${ }^{4}$ Médico assistente voluntário do Departamento de Oftalmologia da Santa Casa de São Paulo. Pós-graduando do Departamento de Oftalmologia do Hospital das Clínicas da Faculdade de Medicina da Universidade de São Paulo.

Endereço para correspondência: Fábio Ejzenbaum, Rua Sergipe, 600 - apto 181 - São Paulo (SP) CEP 001243-000. e-mail:ejz@totalnet.com.br
}

\section{INTRODUÇ̃̃̃O}

A etiologia do estrabismo é assunto abordado e controvertido há mais de 2 séculos, mas até hoje não se chegou a uma conclusão satisfatória, salvo alguns tipos de estrabismo adquiridos, ou congênitos associados a certas síndromes. Mesmo nos casos de esotropia puramente acomodativa, em que a hipermetropia ocupa papel preponderante na sua patogênese, esta não explica por si só o desencadeamento do estrabismo, visto que nem todos os hipermétropes são esotrópicos acomodativos. É claro que tem de haver certa fragilidade do reflexo de fusão nesses pacientes, cuja causa é ainda desconhecida.

Uma das teorias propostas para explicar a patogênese do estrabismo é a refrativa, que teve início com Donders, em $1863^{(*)}$, quando este descreveu pela primeira vez a íntima relação entre a acomodação e a convergência. Donders atribuiu a gênese da esotropia ao excesso de acomodação exigida dos hipermétropes e a da exotropia à insuficiência desta nos míopes. Mas já Donders reconhecia que nem todos os hipermétropes são estrábicos e concluiu que é necessário um fator associado à hipermetropia para que esta possa provocar o aparecimento do estrabismo; identificou a ambliopia como este fator. Já Bielschowsky ${ }^{(1)}$, em 1943, refutava essa teoria, chamando a atenção para casos de esotropia com miopia e exotropia com hipermetropia.

\footnotetext{
* Donders 1863 apud Bielschowsky A Lectures on motor anomalies. Hanover: Dartmouth College Publications; 1943. p. 32
} 
$\mathrm{Javal}^{(2)}$ apresentou a seguinte teoria, para explicar a existência de esotropia em míopes:"...les yeux du myope...converge très fortement pendant la lecture ou la couture et, un beau jour, les muscles droits externes son pour ainsi dire forcés; quand il veux regarder au loin, les myopes vois double et ne peu pas faire rentrer les images l'une dans l'autre." Explicou, em seguida, de outra maneira, a teoria de que os retos laterais são "forçados": existe normalmente uma amplitude de convergência e um percurso de convergência, análogos a uma amplitude de acomodação e um percurso de acomodação. Como o míope olha habitualmente de muito perto, quando sem óculos, torna mais próximo o seu ponto próximo de convergência, e pode ocorrer que, sem nenhuma diminuição da sua amplitude de convergência, seu ponto remoto de visão não esteja mais no infinito, levando ao aparecimento da esotropia. Segundo Javal, esse é o mecanismo da produção do estrabismo convergente dos míopes. Tentando tornar mais clara a teoria de Javal, explicamos o que ele entende por amplitude e percurso. Amplitude de convergência é o tamanho do espaço situado entre o ponto próximo de convergência e a sua máxima divergência; percurso de convergência é a situação espacial desse espaço, o qual pode deslocar-se em bloco, em sentido convergente ou divergente. Exemplificando, imaginemos que um indivíduo possua amplitude de convergência de 30 dioptrias de prisma e que, ao relaxar inteiramente a convergência, tenha os olhos paralelos (ortofórico em posição primária). Seu percurso de convergência situa-se entre o paralelismo ocular e a convergência de 30 dioptrias. Imaginemos, agora, um outro indivíduo, que, relaxando inteiramente sua convergência, tenha os olhos convergentes de 10 dioptrias (esotropia); seu ponto próximo de convergência estaria em 40 dioptrias. Neste caso, o seu percurso de convergência estaria situado entre 10 e 40 dioptrias de convergência.

Embora essas teorias sejam atrativas, não resistem a uma crítica mais profunda. O fato é que a maioria dos estrabismos ainda é considerada "essencial", isto é, não se lhes conhecem as causas. Como não sabemos até onde a miopia ou a hipermetropia fazem parte da etiologia da esotropia, é válido indagar se o mesmo plano cirúrgico age igualmente nos esotrópicos míopes e hipermétropes.

Apesar de menor incidência, a miopia ocorre em pacientes estrábicos, que muitas vezes têm indicação cirúrgica para correção do estrabismo. Entre os pacientes portadores de esotropia, a presença de miopia varia entre $5,2 \%$ e $8 \%^{(3-5)}$. Costenbander ${ }^{(4)}$ e von Noorden ${ }^{(6)}$, estudando pacientes com esotropia congênita, encontraram incidência de miopia entre $5 \%$ e $11 \%$, enquanto os resultados de Shauly et al. ${ }^{(7)}$ revelaram $10,2 \%$.

Almeida et al. ${ }^{(3)}$ avaliaram os resultados dos procedimentos cirúrgicos destinados à correção do estrabismo de 19 pacientes com esotropia e miopia e observaram que diferentes magnitudes de miopia (maior que 1 dioptria), associados a esotropia, não representaram, tanto em termos funcionais como estéticos, fator determinante.

Há um tipo de esotropia, associada a miopias muito altas, geralmente acima de 20 D.E.(dioptrias esféricas), adquirida geralmente na idade adulta, de caráter progressivo, que chega a atingir desvios muito grandes, com forte limitação de abdução e refratária a técnicas operatórias comuns. Knapp denominou esse tipo de estrabismo "esotropia miópica adquirida progressiva"(8)

Teve este trabalho, por objetivo, analisar os resultados cirúrgicos da correção do estrabismo horizontal em portadores de alta miopia, operados no Departamento de Oftalmologia da Santa Casa de Misericórdia de São Paulo, considerando-se alta a miopia maior que 6,0 D.E., excluindo-se, entretanto, a esotropia miópica adquirida progressiva.

\section{MÉTODOS}

Foram avaliados os prontuários de 41 pacientes portadores de alta miopia (maior que $-6,00 \mathrm{DE})^{(9)}$, submetidos a intervenção cirúrgica para correção de estrabismo horizontal no Departamento de Oftalmologia da Santa Casa de Misericórdia de São Paulo, no período de 1972 a 1997, que se enquadravam nos critérios de inclusão.

Foi avaliada a magnitude do desvio na posição primária do olhar, antes e após a operação, utilizando-se os métodos de prisma e "cover" ou Krimsky, segundo o grau de colaboração de cada paciente.

Os pacientes foram divididos em 2 grupos, segundo se tratasse de esotropia permanente ( 24 pacientes) ou exotropia permanente (17 pacientes). Para cada grupo de pacientes com alta miopia, estudou-se um grupo controle de pacientes operados de estrabismo horizontal essencial, não portadores de alta miopia, escolhidos aleatoriamente no fichário.

Os critérios de inclusão neste estudo foram: 1) estrabismo horizontal (eso ou exotropia essencial, sem disfunções de músculos oblíquos); 2) presença de alta miopia em um ou ambos os olhos; 3) não ter sido submetido a correção cirúrgica de estrabismo anteriormente; 4) ausência de alterações orgânicas oculares.

Foram excluídos deste estudo os pacientes esotrópicos com alta miopia que se enquadravam no tipo definido por Knapp como "síndrome da esotropia miópica adquirida e progressiva" ${ }^{(8)}$. Estabeleceu-se como ambliopia a acuidade visual, melhor corrigida opticamente, inferior a 0,6 , ou a diferença de pelo menos duas linhas entre os olhos, com a melhor correção óptica ${ }^{(9)}$.

As características clínicas referentes a idade, sexo, presença ou não de ambliopia, ametropia, desvios pré-operatório e tempo de seguimento encontram-se nas tabelas 1 e 2 .

Todos os planejamentos cirúrgicos obedeceram os critérios rotineiros da Seção de Motilidade Extrínseca do Departamento de Oftalmologia da Santa Casa de Misericórdia de São Paulo e as operações seguiram as técnicas padronizadas na Seção ${ }^{(10)}$. Esse planejamento leva em consideração a presença de ambliopia, hiperfunção de músculos oblíquos e magnitude do desvio na posição primária e nas 4 posições secundárias. Não foi considerada a presença de alta miopia. 
Tabela 1. Características dos pacientes esotrópicos com alta miopia (grupo A) e sem alta miopia (grupo B)

Refração (D.E.)

Desvio pré-operatório $(\Delta)$

Ambliopia tot/uni/bi (N)

Sexo (fem/masc)

Seguimento (meses)

Idade (anos)
Grupo A (N=24)

$-6,00$ a $-29,00$

Média $-12,36 \pm 5,5$

$60,62 \pm 24,46$ (20 a 100)

$24 / 12 / 12$

17 (70,83\%) / 7 (29,16\%)

$14,58 \pm 14,98$ ( 1 a 54 )

$24,47 \pm 12,08$ (5 a 42)
Grupo B ( $\mathbf{N}=28)$

$-0,75$ a $+3,50$

Média $+1,28 \pm 1,17$

$48,93 \pm 18,94$ (23 a 95 )

$15 / 1 / 14$

$13(46,42 \%) / 15$ (53,57\%)

$14,18 \pm 14,95$ ( 1 a 48 )

$17,35 \pm 11,84$ (4 a 48 )

$\mathrm{N}=$ Número de pacientes; D.E. = Dioptrias esféricas; $\Delta$ = Dioptrias prismáticas; tot = total; uni = unilateral; bi = bilateral

\begin{tabular}{|c|c|c|}
\hline & Grupo C (N=17) & Grupo D (N=21) \\
\hline Refração (D.E.) & $\begin{array}{c}-6,00 \text { a }-16,00 \\
\text { Média }-10,12 \pm 2,5\end{array}$ & $\begin{array}{c}-2,00 \text { a }+5,00 \\
\text { Média }+0,5 \pm 0,48\end{array}$ \\
\hline Desvio pré-operatório $(\Delta)$ & $47,52 \pm 12,22(30$ a 70$)$ & $38,00 \pm 12,86$ (20 a 65$)$ \\
\hline Ambliopia tot/uni/bi (N) & $16 / 2 / 14$ & $17 / 5 / 12$ \\
\hline Sexo (fem/masc) & $15(88,23 \%) / 2(11,76 \%)$ & $16(76,20 \%) / 3(23,80 \%)$ \\
\hline Seguimento (meses) & $14,04 \pm 13,60$ (1 a 48) & $11,04 \pm 12,30$ (1 a 47) \\
\hline Idade (anos) & $21,75 \pm 12,24$ (6 a 38) & $21,80 \pm 11,27$ (9 a 44) \\
\hline
\end{tabular}

\section{RESULTADOS}

Os resultados cirúrgicos, de acordo com o tipo de desvio estão apresentados nas tabelas 3, 4 e 5 .

\begin{tabular}{|c|c|c|c|c|c|c|c|c|}
\hline \multicolumn{9}{|c|}{$\begin{array}{l}\text { Tabela 3. Resultado da correção cirúrgica dos pacientes } \\
\text { esotrópicos e exotrópicos altos míopes (grupo I) e não altos } \\
\text { míopes (grupo II). }\end{array}$} \\
\hline & \multicolumn{8}{|c|}{ RESULTADOS } \\
\hline & \multicolumn{4}{|c|}{ ESOTRÓPICOS } & \multicolumn{4}{|c|}{ EXOTRÓPICOS } \\
\hline & $\begin{array}{c}\text { bom } \\
N\end{array}$ & $\underset{N}{\operatorname{mau}}$ & $\begin{array}{c}\text { total } \\
\mathrm{N}\end{array}$ & \%bom & $\begin{array}{c}\text { bom } \\
\mathrm{N}\end{array}$ & $\underset{N}{\operatorname{mau}}$ & $\begin{array}{c}\text { total } \\
\mathrm{N}\end{array}$ & \%bom \\
\hline Grupo I & 8 & 16 & 24 & 33,33 & 10 & 7 & 17 & 58,9 \\
\hline Grupo II & II 20 & 8 & 28 & 71,42 & 15 & 16 & 21 & 71,43 \\
\hline \multicolumn{5}{|c|}{$X^{2}=6,09, P=0,01358$} & \multicolumn{4}{|c|}{$X^{2}=0,70, P=0,4028$} \\
\hline
\end{tabular}

Tabela 4. Tipos de maus resultados pós-operatórios dos pacientes altos míopes (grupo I) e não altos míopes (grupo II)

\section{RESULTADOS}

\begin{tabular}{cccc}
\hline \multicolumn{4}{c}{ ESOTRÓPICOS } \\
\hline Sub & Super & Total \\
N $\quad \%$ & N $\%$ &
\end{tabular}

$\begin{array}{llllllllllll}\text { Grupo I } & 15 & 93,7 & 1 & 6,3 & 16 & 6 & 85,7 & 1 & 14,3 & 7\end{array}$

$\begin{array}{lllllllllll}\text { Grupo II } & 7 & 87,5 & 1 & 12,5 & 8 & 6 & 100 & 0 & 0 & 6\end{array}$

$\begin{array}{llll}\text { Total } 22 & 2 & 12\end{array}$

$\mathrm{N}=$ Número de pacientes. Sub $=$ subcorrigidos. Super $=$ supercorrigidos

\begin{tabular}{|c|c|c|c|c|}
\hline \multicolumn{5}{|c|}{$\begin{array}{l}\text { Tabela 5. Valores das correções cirúrgicas dos pacientes } \\
\text { esotrópicos e exotrópicos,altos míopes (grupo I) e não altos } \\
\text { míopes(grupo II) }\end{array}$} \\
\hline & \multicolumn{4}{|c|}{ RESULTADOS } \\
\hline & \multicolumn{2}{|c|}{ ESOTRÓPICOS } & \multicolumn{2}{|c|}{ EXOTRÓPICOS } \\
\hline & $\Delta$ & $\%$ & $\Delta$ & $\%$ \\
\hline Grupo I & $44,25 \pm 23,60$ & $71,80 \pm 32,10$ & $38,20 \pm 14,31$ & $80,24 \pm 20,17$ \\
\hline Grupo II & $43,04 \pm 20,52$ & $86,04 \pm 23,32$ & $29,05 \pm 14,98$ & $75,77 \pm 21,70$ \\
\hline $\mathrm{P}$ & 0,843470 & 0,070474 & 0,071469 & 0,833649 \\
\hline \multicolumn{5}{|c|}{$\Delta=$ Correção absoluta, $\%=$ Correção percentual } \\
\hline
\end{tabular}

\section{COMENTÁRIOS}

O estudo demonstrou que, nos pacientes esotrópicos altos míopes, a porcentagem de bons resultados foi estatisticamente menor do que no grupo de pacientes sem alta miopia (Tabela 3 - $\mathrm{P}=0,01358$ ). Entre os 16 pacientes altos míopes com maus resultados, 15 ficaram sub-corrigidos (Tabela 4) e apenas 1 evoluiu com supercorreção. Observou-se que, dos 8 pacientes esotrópicos sem alta miopia e com mau resultado cirúrgico, 7 ficaram subcorrigidos (Tabela 4). Quanto à correção absoluta do desvio, observou-se semelhança estatística entre os grupos com e sem alta miopia (Tabela 5 - $\mathrm{P}=0,84347$ ); com a magnitude das amostras estudadas, a correção percentual não mostrou diferença estatisticamente significante entre os dois grupos (Tabela 5 - $\mathrm{P}=0,070474$ ). Esse resultado, se levados em consideração os valores dos desvios préoperatórios, pode ser considerado clinicamente importante.

O mau resultado cirúrgico nos altos míopes esotrópicos, 
observado neste trabalho, coincide com outros estudos, que comentaremos adiante.

Quanto ao trabalho de Almeida e et al. ${ }^{(3)}$, não é possível estabelecer comparação com esta pesquisa, visto haverem eles estudado pacientes com miopia a partir de 1 D.E.

Shauly e et al. ${ }^{(7)}$ mostraram que, em pacientes portadores de esotropia congênita com alta miopia, entre $-8,00$ e $-19,50$ D.E., obtiveram $100 \%$ de subcorreção cirúrgica; em $71 \%$ deles, o desvio residual foi maior que $20 \Delta$, freqüencia três vezes maior do que no grupo sem alta miopia, tanto a curto ( 6 meses) como a longo prazo (4 anos). A explicação que propõem para esse fato é que, em olhos com grande comprimento axial, existiria relação inversa com o bom resultado pós-operatório, ou seja, olhos de maior diâmetro ântero-posterior têm menor resposta à mesma magnitude de retrocesso-ressecção do que olhos com comprimento axial normal, talvez por esse olhos ficarem comprimidos na cavidade orbitária.

Ruttum et al. ${ }^{(11)}$ observaram, através de ultra-sonografia, em um paciente anisometrópico com esotropia (olho direito $=-23$ D.E. $=-5,00$ D.C. $90^{\circ}$, diâmetro $=32 \mathrm{~mm}$, e olho esquerdo $=-4,75$ D.E. $=-1,75$ D.C. $10^{\circ}$, diâmetro $=25,60 \mathrm{~mm}$ ), contato do bulbo ocular com a parede medial orbitária durante a abdução, somente do olho direito, mais míope, o qual apresentava limitação de abdução; isso sugeriria, segundo os autores, ser este o fator determinante do desvio apresentado pelo paciente.

Demer e et al..$^{(5)}$, estudando um paciente alto míope esotrópico com comprimento axial aumentado (olho direito $=34,5 \mathrm{~mm}$, olho esquerdo $=32,5 \mathrm{~mm}$ ), observaram, através de tomografia computadorizada (TC), que os olhos encontravam-se em grande proximidade com as paredes orbitárias, estabelecendo-se contacto entre eles e as paredes laterais destas.

Bagolini et al. ${ }^{(12)}$, estudando 6 pacientes com "síndrome da esotropia miópica adquirida progressiva", notaram que todos os olhos tinham comprimeto axial aumentado e alta miopia. Os pacientes foram submetidos a TC e ecografia, através do que mediram os espaços entre a parede orbitária lateral e o bulbo. Observaram que a distância era muito pequena e que, à adução, havia contacto entre o reto lateral (RL) e aquela parede. Sugeriram que essa compressão, com o tempo, poderia levar à hipo-atividade deste músculo, seja por atuação sobre os capilares, fibras nervosas ou as próprias fibras musculares, provocando, com isso, a esotropia.

Meyer et al. ${ }^{(13)}$ estudaram, através de microscopia eletrônica, retos laterais hipofuncionantes de 4 pacientes altos míopes esotrópicos (três com esotropia congênita e um com esotropia adquirida, com miopias entre-7,00D.E. e-10,00D.E.). Nos três pacientes com esotropia congênita, o RL encontrava-se totalmente fibrosado; no paciente com esotropia adquirida, aproximadamente $80 \%$ das fibras musculares apresentavam fibrose e, nas $20 \%$ restantes, observava-se algum grau de degeneração. Os autores propuseram a hipótese de que o processo de fibrose do RL, nestes pacientes, esteja relaciona- do à idade de aparecimento do desvio, já que a degeneração antecede a fibrose. Infelizmente, os autores não referiram o comprimento axial dos olhos desses 4 pacientes.

Estudos histológicos do reto lateral de pacientes esotrópicos míopes revelaram, através de microscopia eletrônica, presença de fibrose e aumento do tecido conectivo intersticial, com espessamento da membrana basal do endotélio capilar $^{(7,12-13)}$. A redução da função muscular, associada à miopia progressiva, demonstrada nesses trabalhos, sugere a hipótese de tratar-se de paresia miogênica.

Entretanto, apesar dos estudos, acima apresentados, através de imagem, relativos à compressão do RL contra a órbita e estudos histológicos revelando alterações estruturais musculares, os resultados demonstraram que a porcentagem de subcorreção foi semelhante entre os pacientes esotrópicos com alta miopia e sem alta miopia.

Entre os pacientes exotrópicos deste estudo, não se evidenciaram diferenças de resultados estatisticamente significantes entre os grupos com e sem alta miopia, e não encontramos, na literatura, nenhum estudo fazendo menção à diferença, nos resultados cirúrgicos, entre exotrópicos altos míopes e não altos míopes.

Este trabalho demonstrou que os pacientes esotrópicos com alta miopia (>-6,00 DE), apresentaram maior incidência de maus resultados em comparação com pacientes esotrópicos com erro refrativo entre $-0,50 \mathrm{DE}$ e $+3,50 \mathrm{DE}$.

\section{ABSTRACT}

Purpose: To analyze results of correction of horizontal strabismus surgical in high myopia patients. Methods: A retrospective chart review of 24 patients with esotropia and high myopia (greater than -6.00 spherical diopters) and 17 patients with exotropia and high myopia compared with their control groups. Deviation between $10 \Delta$ of esotropia and $10 \Delta$ of exotropia was considered a surgical success. Results: We noticed a high incidence of bad results among the esotropic patients with high myopia. Conclusion: There is a tendency of bad results among the esotropic patients with myopia greater than -6.00 when compared with patients with spherical refractive error between -0.75 and +3.50 .

Keywords: Strabismus/surgery; Myopia; Exotropia; Esotropia; Surgery

\section{REFERÊNCIAS}

1. Belschowsky A. Lectures on motor anomalies. Hanover: Darthmouth College; 1943. p. 32-3.

2. Javal E. Manual du strabisme. Paris: Masson; 1986. p. 174-5.

3. Almeida H, Jampolsky A, Fitton M. Surgical results in esotropia associated with myopia. Am J Ophthalmol 1965;60:448-52.

4. Costenbander FD. Infantile esotopia. Trans Am Ophthalmol Soc 1961;59:397.

5. Demer JJ, Von Noorden GK. High myopia as an unusual case of restrictive motility disturbance. Surv Ophthalmol 1989;33:281-4.

6. Von Noorden GK. Esodeviations. In: Von Noorden GK. Binocular vision and ocular motility: theory and management of strabismus. St Louis: Mosby; 1990. p. 295. 
7. Shauly Y, Miiller B, Meyer E. Clinical characteristics and long-term postoperative results of infantile esotropia and myopia. J Pediatr Ophthalmol Strabismus 1997;34:357-64.

8. Knapp P. Surgical result of esotropia associated with myopia. [Apresentado 1a. Reunião dos Discípulos de Ciência; 1990 maio 18-22; Foz de Iguaçu].

9. Rosenfield M. Miopia. In: Benjamin WJ. Borish's clinical refraction. Philadelphia: Saunders; 1998. p.3-5.

10. Prieto-Diaz J, Souza-Dias C. Estrabismo. 3ed. Argentina: La Plata; 1996. p. 558-70.
11. Ruttum MS, Lloyd MA, Lewandowsky MF. Ecography in the diagnosis of restrictive motility caused by severe myopia. Am J Ophthalmol 1990; 109:350-1.

12. Bagolini B, Tamburelli C, Dickmann A, Colosino C. Convergent strabismus fixus in high myopic patients. Doc Ophthalmol 1990;74:309-20.

13. Meyer E, Ludatscher RM, Lichtig C, Shauly Y, Gdal-On M. End stage fibrosis of The lateral rectus muscle in myopia with esotropia. Ophthalmic Res 1990;22:259-64.

\section{1 - FÓRUM NACIONAL dE SAÚde OCULAR}

\section{E 31 DE MAIO DE 2001}

\section{REALIZAÇÃO: \\ Conselho Brasileiro de Oftalmologia e Senado Federal}

Local: Auditório Petrônio Portella

Senado Federal - Brasília - DF

- Promoção de palestras sobre os avanços da Oftalmologia Brasileira no campo científico e de ensino, na promoção da saúde ocular da população e na atuação assistencial

- Apresentação de exposição de trabalhos premiados e painéis sobre a especialidade.

\section{Inscrição: tel/fax (0xx61) 327-1606 \\ e-mail: cbobsb@terra.com.br}
As inscrições são gratuitas, mas o número de vagas limitado, por determinação da Segurança do Senado Federal

